\title{
ALGUNAS POSIBILIDADES DELARTÍCULO DE INVESTIGACIÓN COMO RECURSO DIDÁCTICO ORIENTADO A CUESTIONAR IDEAS INADECUADAS SOBRE LA CIENCIA
}

\author{
Campanario, Juan Miguel \\ Departamento de Física. Universidad de Alcalá. 28871 Alcalá de Henares. Madrid \\ juan.campanario@uah.es \\ http://www.uah.es/otrosweb/jmc
}

\begin{abstract}
Resumen. En este trabajo se propone el uso de los artículos científicos como un recurso para la enseñanza de las ciencias. Se analizan las posibilidades didácticas de los artículos científicos y se sugieren formas de utilizar este recurso para cuestionar ideas inadecuadas sobre la ciencia y el conocimiento científico.
\end{abstract}

Palabras clave. Enseñanza de las ciencias, concepciones epistemológicas, artículo científico.

Summary. In this paper I suggest the use of scientific articles as a tool in science teaching. I analyse the didactic possibilities of scientific articles and I suggest some ways to use this resource to challenge some inadequate ideas about science and scientific knowledge.

Keywords. Science teaching, epistemological conceptions, scientific article.

\section{INTRODUCCIÓN}

Uno de los objetivos generales de la enseñanza obligatoria de las ciencias es que los futuros ciudadanos educados desarrollen ideas adecuadas sobre los procesos de construcción y justificación del conocimiento científico. Por otra parte, la enseñanza de las ciencias en la universidad debería preparar al futuro licenciado para poder dedicarse, en principio, a tareas generales de investigación en el marco de un programa de formación (doctorado) o para el desempeño de tareas profesionales en una industria, en un centro de investigación o en la docencia en distintos niveles. Existen indicios suficientes en la literatura educativa de que tanto en un caso como en otro, las ideas del alumnado sobre los procesos de construcción de la ciencia pueden ser inadecuadas.

En los últimos años la investigación educativa ha comenzado a prestar especial atención a uno de los factores que plantea problemas y dificultades en el aprendizaje de las ciencias: las concepciones epistemológicas del alumnado. Las concepciones epistemológicas se refieren a las ideas sobre la ciencia y el conocimiento científico, así como a las ideas sobre su construcción, evolución y fundamentación (Ryan y Aikenhead, 1992; Gaskell, 1992; Wolff-Michael, 1994; Campanario y Otero, 2000; Campanario, 2002c). Como es sabido, el profesorado de ciencias, los estudiantes de enseñanza secundaria y de universidad con frecuencia mantienen ideas inadecuadas sobre los aspectos citados. Entre las concepciones epistemológicas comunes que se pueden identificar cabe citar la creencia tan extendida de que el conocimiento científico está probado más allá de cualquier duda o la idea de que la ciencia es una actividad en continuo avance (sin errores o reformulaciones conceptuales). Otras concepciones muy extendidas hacen referencia a la supuesta neutralidad y asepsia del conocimiento científico, que tendría su origen en el uso de un «método» 
mediante el que se obtiene, casi como con una receta, el resultado correcto y, por tanto, indiscutible. Desde estas concepciones ingenuas, el papel de los investigadores se asimila más al de meros descubridores de la verdad, que al de constructores de teorías tentativas. En un trabajo anterior hemos analizado con más detalle el papel de las concepciones epistemológicas de los estudiantes como un obstáculo para el aprendizaje de las ciencias (Campanario y Otero, 2000). El lector interesado puede encontrar allí más información sobre este tema. A la vista de los problemas relacionados con los factores citados, los nuevos puntos de vista en didáctica de las ciencias prestan especial atención a los procesos de investigación científica y se reconoce la conveniencia de acercar al alumnado a la realidad del trabajo de los científicos. En este empeño, la filosofía de la ciencia ha sido una fuente continua de inspiración en nuestra área.

Desgraciadamente, las teorías dominantes en filosofía de la ciencia que intentan explicar los procesos de construcción y justificación del conocimiento científico están lejos de ser unánimes. No existe, por tanto, un punto de vista aceptado de manera general que permita tomar decisiones generales acerca de la visión «adecuada» que nuestro alumnado debería desarrollar sobre la actividad científica. Además, como hemos argumentado en un trabajo anterior (Campanario, 1999), las teorías en filosofía de la ciencia se basan generalmente en el análisis de episodios especialmente relevantes en la historia de la ciencia (el advenimiento de la nueva física, la mecánica cuántica, etc.). Si fijamos nuestra atención únicamente en momentos históricos tan destacados y trascendentales como los anteriores, corremos un cierto peligro de olvidar la realidad del trabajo de los miles de científicos que, sin poner en peligro los paradigmas existentes, contribuyen cada día a la construcción de nuevo conocimiento y al desarrollo tecnológico. En otros casos podemos encontrar científicos que cuestionan los paradigmas reinantes cuando no existe ninguna crisis (Campanario, 2003; Campanario y Martin, 2003). Ciertamente, muchas de las descripciones que se nos ofrecen en filosofía de la ciencia tienen poco que ver con la realidad cotidiana de un departamento universitario o de un centro de investigación.

En este artículo nos proponemos seguir desarrollando una línea de actuación docente orientada a favorecer el desarrollo de ideas y concepciones adecuadas sobre los procesos científicos. Se trata de acceder, en la medida de lo posible, al trabajo diario de los investigadores y a los factores que condicionan su quehacer. Precisamente, uno de los factores que más influyen en la construcción del conocimiento por parte de los investigadores es la comunicación con otros colegas, dado que, de la opinión de estos últimos, depende el éxito o el fracaso final del trabajo propio.

El papel de la comunicación del conocimiento resulta especialmente relevante en nuestros días, ya que en los últimos años se están desarrollando canales alternativos (p.e., revistas electrónicas, grupos de discusión en internet...) que facilitan la difusión de las contribuciones académicas. A pesar de la influencia de la comunicación científica en las decisiones y estrategias que desarrollan los investigadores, éste es uno de los elementos a los que menos atención se presta en filosofía de la ciencia y, como consecuencia, en la presentación que hacemos de la ciencia al alumnado y en las visiones que transmitimos.

Las razones anteriores explican que en este trabajo nos centremos en el estudio del medio fundamental en la comunicación científica: el artículo de investigación. La idea que rige nuestra propuesta es sencilla: los artículos que publican los investigadores pueden ayudar al profesorado a presentar una visión más cercana a la realidad de los procesos de construcción y justificación de las disciplinas, a la vez que permiten cuestionar algunas concepciones epistemológicas inadecuadas. Por tanto, los objetivos que perseguimos al plantear el papel del artículo científico como un nuevo recurso docente son:

a) Facilitar el desarrollo de ideas adecuadas sobre la ciencia y el conocimiento científico.

b) Analizar aspectos generales relacionados con la estructura y las estrategias argumentativas que son comunes en los artículos científicos de cara a la consecución del objetivo anterior.

c) Analizar algunos apartados concretos de los artículos científicos y discutir estrategias de trabajo en el aula, orientadas al logro del primer objetivo.

Aunque es evidente que el alumnado de enseñanza secundaria, e incluso de universidad, no va a poder entender el contenido de casi ningún artículo científico como los que se publican habitualmente en revistas profesionales de física o química, es posible aprender mucho acerca de la dinámica de la ciencia si miramos con otros ojos la estructura de un trabajo de investigación y si prestamos atención a determinados aspectos que pueden pasar desapercibidos a primera vista. Evidentemente, para poder profundizar en los argumentos concretos y en la exposición que se realiza en un artículo de investigación es preciso estar familiarizado con el tema (por esta razón resulta, a veces, tan difícil encontrar revisores capaces de entender artículos en un tema determinado). Lo que planteamos aquí no es una lectura en profundidad de un artículo, sino, en la mayor parte de los casos, la búsqueda en un artículo de investigación de indicios y señales que pongan de manifiesto la realidad de algunas de las posibles estrategias argumentativas que se utilizan en la ciencia. En esta búsqueda, la orientación por parte del profesorado es fundamental.

Es necesario, por otra parte, tener en cuenta que los artículos que se publican en revistas correspondientes a disciplinas distintas pueden tener diferencias importantes. No es lo mismo un artículo de física experimental que uno de biología o de química. Es preciso, por tanto, prestar atención a estas diferencias cuando se utiliza un artículo de investigación como instrumento didáctico. Por esta razón resulta difícil ofrecer sugerencias generales válidas en todos los casos.

El resto del trabajo se organiza en cuatro apartados. En primer lugar se abordan los aspectos generales de un ar- 
tículo científico que se pueden analizar en clase. A continuación, profundizamos en el papel que desempeñan algunas secciones concretas de los artículos y discutimos la utilidad que pueden tener para fomentar un debate sobre distintos aspectos relacionados con la construcción y justificación del conocimiento científico. En otra sección se ofrecen sugerencias concretas sobre cómo utilizar los artículos de investigación en el aula. Por último, se discuten las posibles ventajas del enfoque que proponemos y se plantean algunas conclusiones generales.

Los profesores y profesoras que quieran utilizar este recurso pueden conseguir artículos de investigación en cualquiera de las revistas científicas que se publican en español. También es posible conseguir estos documentos en internet. Es posible que una parte de las actividades que se presentan en este trabajo sólo puedan realizarse con alumnado universitario o con profesorado de secundaria en formación en los cursos del CAP o del CCP. Nosotros, por ejemplo, hemos utilizado los artículos científicos como un recurso en una asignatura optativa llamada «Aprendizaje de las Ciencias Experimentales» que se ofrece en $4^{\circ}$ curso de la licenciatura de Química en la Universidad de Alcalá, para aquellos alumnos y alumnas que puedan tener interés en dedicarse a la docencia en el futuro.

\section{ANÁLISIS DIDÁCTICO DE LOS ASPECTOS GENERALES DE LOS ARTÍCULOS CIENTÍ- FICOS}

\section{Existen diferentes clases de artículos científicos}

Basta examinar con nuestros alumnos varios números de cualquier revista académica para detectar fácilmente diferentes tipos de artículos. Los más comunes son:

a) Artículo de investigación: en ellos se describe un trabajo completo de investigación o una parte del mismo.

b) Revisión: se analiza críticamente el estado del conocimiento en un área o un tema concreto a partir de la bibliografía publicada.

c) Retracción: un autor corrige o retira un trabajo propio anterior debido a factores diversos (imposibilidad para replicar los resultados, denuncias de fraude, errores, etc.).

d) Comentarios y críticas: como su nombre indica, un autor comenta o critica un trabajo anterior publicado por otros investigadores.

e) Artículo teórico: se plantea un modelo, una teoría o un sistema para entender un fenómeno o conjunto de fenómenos, una realidad concreta o un dominio de conocimientos.

En este trabajo nos centraremos en el uso didáctico de los artículos de investigación.

\section{Los artículos de investigación tienen una estructu- ra característica}

Los artículos en los que se describe un trabajo de investigación tienen una estructura general más o menos estandarizada si bien existen algunas ligeras diferencias entre disciplinas. Esta estructura sirve de molde mental en el que encaja el contenido del artículo y, por tanto, ayuda al lector a encontrar la información relevante de acuerdo con sus intereses (p.e., metodología, referencias citadas, marco teórico, etc.).

Naturalmente, la estructura típica de un artículo no refleja, en general, el modo en que se realizó la investigación. Como dijo Feynman en la conferencia que pronunció con motivo de la recepción del premio Nobel: «[...] tenemos la costumbre de escribir los artículos que se publican en las revistas científicas de manera que el trabajo aparezca tan acabado como sea posible, para tapar todos los otros caminos y no tener que preocuparnos de explicar los intentos fallidos ni tener que describir cómo la primera idea que se nos ocurrió era incorrecta» (Feynman, 1965). Es decir, aunque es razonable esperar que en un artículo científico se nos cuente la verdad, no podemos esperar toda la verdad.

Un profesor o profesora de ciencias no debería tener ningún problema en identificar los distintos apartados de un artículo de investigación y en explicar al alumnado el papel de cada uno de dichos apartados: título, autores, resumen (abstract), palabras clave (keywords), introducción, objetivos, métodos, resultados, discusión, conclusiones, agradecimientos, referencias, anexos (si los hay). Por esta razón no nos detenemos a explicar con detalle su contenido.

\section{Los artículos de investigación abordan un tema muy limitado}

Es posible que, cuando nuestro alumnado se enfrente por primera vez a un artículo de investigación, quede muy sorprendido al descubrir las notables diferencias que existen entre la forma y el contenido del mismo y la forma y el contenido de sus libros de texto (la única prosa científica a la que, probablemente, haya tenido acceso anteriormente). Algo que suele llamar bastante la atención es la especificidad del contenido: no es necesario ser un experto para darse cuenta de que la mayor parte de los artículos abordan temas muy reducidos y limitados, a diferencia de las grandes teorías y paradigmas que se presentan en los libros de texto de ciencias. Además, es fácil detectar la ausencia casi total de los nombres « $f a-$ mosos» que han acompañado al estudiante hasta ahora (Newton, Einstein, Arrhenius, etc.) que son sustituidos por otros totalmente desconocidos (Anderson, Kintsch, etc.). Todos los indicios hacen ver al neófito que se está adentrando en un terreno muy especializado en el que sus conocimientos generales resultarán de poca utilidad. Una primera conclusión interesante es que la mayor parte del trabajo científico «normal» se centra en aspectos muy limitados dentro del marco general de un paradigma que se da por supuesto. 


\section{Los artículos de investigación permiten detectar vi- siones inadecuadas sobre el «método científico»}

Una de las quejas más comunes de los investigadores en nuestra área se refiere al modo tradicional en que se introducen los contenidos en los manuales escolares de ciencias. Por ejemplo, es muy frecuente que los conceptos se presenten como el resultado casi inmediato o evidente de unas cuantas observaciones y deducciones (Otero, 1985). Sirva, como ejemplo, la forma tradicional de introducir los conceptos de carga eléctrica (a partir de unas cuantas experiencias de atracción o repulsión entre objetos) o de ácido y base (a partir de unos supuestos cambios de color de un «papel de tornasol» del que no se había oído hablar antes). No resulta raro que estas pautas tradicionales, sumamente simplificadas e irreales, refuercen visiones inductivistas ingenuas acerca de los procesos de construcción del conocimiento científico.

Por otra parte, muchos libros de texto incluyen versiones idealizadas de un supuesto «método científico» que se aplicaría de manera casi automática y general para «descubrir» los conocimientos y las leyes naturales. Según parece, este supuesto «método científico» consta de una serie de fases bien conocidas, como son la observación desprovista de toda teoría, la obtención de datos, la generación de una hipótesis y su posterior comprobación que, aparentemente, es definitiva. Sin embargo, cuando se analiza un artículo de investigación, resulta evidente que la estructura del mismo no se corresponde con las fases del supuesto «método científico» general al que aludimos; muy al contrario, del análisis general de un artículo se desprenden algunas consecuencias interesantes sobre el proceso de construcción del conocimiento científico que plantean dudas casi inmediatas sobre la existencia del famoso método. Es posible analizar en clase, con nuestros alumnos, los siguientes aspectos que caracterizan la prosa típica de los artículos de investigación:

a) Continuidad: En general, el trabajo de investigación que se realiza suele ser continuación o ampliación de uno previo. Esto se aprecia más claramente en las citas y referencias.

b) Papel dominante de la teoría: una teoría o un enfoque general dirigen las mediciones y observaciones, lo cual contrasta con las visiones inductivistas ingenuas, que enfatizan el papel de la observación desprovista de expectativas previas como punto inicial del proceso de indagación.

c) Carácter tentativo: las conclusiones que se obtienen suelen ser tentativas y es común encontrar discusiones sobre alternativas y propuestas de futuros trabajos de ampliación: lejos, todo ello, de la confirmación definitiva de las hipótesis (última fase del supuesto «método científico»)

Por otra parte, el análisis de un artículo de investigación puede ayudarnos a cuestionar la creencia ampliamente extendida (incluso entre los propios investigadores) de que la tarea de los científicos consiste fundamentalmente en contrastar las teorías con la experiencia intentando de encontrar discrepancias que permitan descartarlas y cambiarlas por otras mejores. Este punto de vista (falsacionismo ingenuo) tiene a Popper como su principal defensor, si bien hoy día se considera que representa una visión inadecuada sobre la ciencia y la evolución del conocimiento científico (Chalmers es autor de un análisis detallado de las debilidades del falsacionismo (Chalmers, 1982)); muy al contrario, si leemos con cierto detalle el contenido de los artículos de investigación, podremos comprobar que la mayoría de los trabajos tienen como objeto confirmar teorías, en vez de falsarlas. De hecho, la tarea que se realiza consiste, fundamentalmente, en lo que Kuhn (1971) describe como «completar el paradigma». Así, en la inmensa mayoría de los artículos se describen trabajos en los que se llevan a cabo cálculos de parámetros físicos, síntesis de compuestos, diseño de nuevos métodos para obtener determinados resultados, etc. Todo ello se hace dentro del marco general de una teoría aceptada (y no cuestionada) y, por supuesto, no tanto con la idea de falsarla como con la intención de confirmarla y completarla y no entrar en conflicto con los postulados básicos que guían la labor realizada, algo que puede acarrear graves problemas al investigador (Campanario, 2003).

Es posible, sin embargo, encontrar artículos en los que se describe un conflicto entre alguna teoría o una parte de ella (p. e., una ecuación) y los datos experimentales obtenidos. En estos casos, en contra de lo que cabría esperar, si la ciencia funcionase mediante la falsación simple, no se rechaza automáticamente la teoría. Según Chinn y Brewer, una respuesta posible consiste en mantener los datos en una especie de «cuarentena» e incluso en rechazar o negar los datos (Chinn y Brewer, 1993).

\section{¿Cuántos artículos escriben los científicos?}

En alguna ocasión hemos tenido que responder a una pregunta tan directa como la anterior. Dicha pregunta puede formularse de otra manera: ¿cuál es la productividad media de los científicos? Ésta es una interesante cuestión que puede surgir en cualquier momento y que no tiene una respuesta fácil o unívoca. Para alguien, como cualquiera de nuestros alumnos o alumnas, que encuentra cada día en los periódicos y revistas generales artículos escritos por los mismos autores, puede resultar sorprendente enterarse de que un trabajo de tesis que se prolonga por cuatro, cinco o más años puede dar lugar, en el mejor de los casos, a tres o cuatro artículos. Cuando explicamos a un lego que una productividad de cuatro artículos en diez años en las revistas incluidas en las bases de investigación de los índices de citas SSCI es alta en nuestra área (según se deduce de un estudio de Fernández-Cano, 1999), puede conseguir una impresión más adecuada acerca de la dificultad inherente a la generación de nuevo conocimiento.

\section{La necesidad de justificar la investigación}

Es frecuente que en los artículos científicos se detallen las razones que justifican la investigación que se describe en ellos. Estas justificaciones pueden aparecer 
en diferentes apartados, pero son más comunes en la introducción (se indica qué problema se trata de resolver) o en la sección de conclusiones. Normalmente estas justificaciones hacen referencia a los aspectos positivos del trabajo, a las posibles aplicaciones o continuaciones y a las respuestas que ofrece a las preguntas conceptuales que guían la investigación. La necesidad de explicar por qué un trabajo es interesante para quienes lo realizan o útil para la comunidad académica o para la sociedad es algo que puede, de nuevo, resultar llamativo cuando el alumnado tiene un primer contacto con un artículo de investigación. No en vano, una de las concepciones tradicionales sobre la ciencia es que ésta es una actividad que busca la verdad por encima de todo de manera desinteresada y ésa debería ser, aparentemente, una motivación suficiente. De nuevo, tenemos un pretexto para insistir en clase en los aspectos sociales de la construcción del conocimiento científico. Parafraseando a Borges, podríamos afirmar, casi, que el trabajo del científico consiste tanto en presentar sus «descubrimientos» como en explicar por qué éstos son memorables.

\section{La novedad del contenido de un artículo es un factor decisivo en su aceptación}

Según una concepción común ampliamente extendida, en la ciencia ningún resultado se considera definitivo hasta que no ha sido replicado por otro investigador independiente. Sin embargo, basta realizar una pequeña exploración de una revista profesional o de una base de datos que incluya los abstract (o resúmenes de los artículos), para darse cuenta de que muy pocos trabajos se orientan a replicar, sin más, estudios anteriores (Campanario, 1999). Muy al contrario, la novedad se suele considerar un mérito en el entorno académico. Las propias revistas académicas suelen reflejar este deseo de novedad en las directrices para los autores. Un examen atento de los artículos científicos pone de manifiesto cómo los investigadores se esfuerzan precisamente en destacar los aspectos novedosos de su tarea, bien sea en el enfoque utilizado, en el método seguido o en las conclusiones alcanzadas.

Por otra parte, los investigadores que estudian la dinámica de la ciencia llaman la atención sobre la dificultad real de replicar trabajos anteriores debido a la enorme cantidad de conocimiento tácito que desarrollan los científicos sobre un tema o un procedimiento determinado y las dificultades para expresar y formalizar este conocimiento tácito (Collins, 2001; Polanyi, 1958). Por ejemplo, no es raro descubrir diferencias más o menos importantes en dos experimentos aparentemente similares. La dificultad de una replicación total puede apreciarse mejor durante el transcurso de una controversia científica. En estos episodios suele haber más intentos de replicación de trabajos anteriores y la sección de «métodos» de los artículos que se publican adquiere una importancia fundamental (Collins y Pinch, 1993). Los investigadores que han estudiado la evolución de las controversias científicas destacan las enormes dificultades que tienen los contendientes para ponerse de acuerdo acerca de si un investigador ha logrado replicar realmente el trabajo de otro o más bien faltan o sobran detalles que son importantes y que explican por qué en una determinada repetición de un experimento no se obtuvieron los mismos resultados que en el estudio original (Collins y Pinch, 1993). Estos detalles pueden ilustrarse con una sencilla actividad en clase orientada a analizar una posible replicación de una experiencia haciendo hincapié en la cantidad de factores que pueden hacer que dos replicaciones aparentemente iguales den resultados diferentes.

\section{Los autores del artículo guían al lector por el ca- mino deseado}

Una de las características típicas de los libros de texto de ciencias es su estilo impersonal. A diferencia de otros textos expositivos, en los manuales escolares actuales se suele utilizar la tercera persona o la voz pasiva, si bien en el pasado fueron comunes otros formatos en los libros de ciencias (Strube y Lynch, 1984). Por otra parte, las guías sobre cómo escribir y publicar trabajos de investigación, insisten en el carácter impersonal y aséptico de la prosa científica y aconsejan al autor de un artículo que no se implique personalmente en su desarrollo. Sin embargo, un análisis cuidadoso del estilo de los artículos de investigación revela algunas características que permiten descubrir la presencia sutil de los autores. Estos rasgos de estilo contrastan fuertemente con la prosa habitual en los libros de texto de ciencias, por lo que conviene insistir ante nuestro alumnado para que tenga presente estas diferencias.

Una de las formas en que los autores se implican es mediante determinados juicios valorativos acerca del interés del trabajo o acerca del carácter inesperado y sorprendente de algún resultado. Igualmente, los autores de los trabajos de investigación suelen expresar su opinión acerca de las dificultades encontradas o acerca de posibles interpretaciones. Estas opiniones se traducen, indirectamente, en una determinada valoración del trabajo realizado. Por ejemplo:

- Los resultados de los espectros son difíciles de interpretar.

- Podríamos pensar a primera vista que ...

- Un examen más detenido de los datos revela que...

Otra estrategia que utilizan los investigadores para hacer notar su presencia es el uso de instrucciones o directivas que orientan y conducen al lector. En los últimos años los expertos en lingüística han empezado a estudiar este tipo de recursos pragmáticos y el papel que desempeñan en la defensa de una determinada línea argumentativa por parte de los científicos (Hylan, 2002). En los artículos de investigación es fácil identificar instrucciones directas orientadas a conseguir que el lector haga algo de determinada forma, preste atención a determinados aspectos o piense de la forma que el autor desea. Los siguientes ejemplos ilustran estas situaciones:

\section{- Considérese la posibilidad siguiente..., \\ - Compárense en primer lugar las tablas III y IV... \\ - El primer factor al que debemos prestar atención es...}




\section{- Debido a las limitaciones del método, es necesario tener en cuenta que...}

El uso de este tipo de recurso estilístico y pragmático es más común de lo que pudiera pensarse inicialmente $\mathrm{y}$, en definitiva, nos debería hacer recapacitar sobre la supuesta neutralidad del lenguaje científico. Frente a esta supuesta neutralidad, desde diversos puntos de vista se destaca el carácter interactivo del discurso típico que se utiliza en los artículos, como un enfoque al servicio de la necesidad de convencer al lector. Insistiremos más en este aspecto más adelante (nótese la implicación del autor).

La presencia de los autores del artículo se puede detectar fácilmente también cuando éstos admiten su ignorancia o reconocen problemas o limitaciones en los procedimientos seguidos. Sirvan, como ejemplo, las expresiones siguientes:

- No conocemos ningún trabajo que aborde de manera sistemática el cálculo de...

- Sospechamos que la membrana puede ser permeable a determinados solutos...

- Hasta ahora no hemos encontrado otra explicación a la anomalía que aparece en los datos...

Los investigadores suelen incluir, asimismo, algunos comentarios valorativos sobre trabajos y estudios realizados por otros, por ejemplo:

- Aunque X afirmó haber obtenido... otros critican sus resultados por...

Aunque, efectivamente, el lenguaje propio de la ciencia es más aséptico e impersonal que el de otras disciplinas, los autores no desaparecen del todo: están allí argumentando sutilmente y orientando al lector para que siga el camino que conduce a las interpretaciones que ellos desean transmitir. Nuestro alumnado puede buscar ejemplos similares.

\section{El resultado del trabajo científico se presenta de manera tentativa}

Una característica notable de los artículos científicos es la cautela con la que los investigadores presentan sus resultados y conclusiones. Este rasgo contrasta fuertemente con la rotundidad y seguridad absoluta con que se plantean los contenidos en los libros de texto de ciencias, algo a lo que nuestro alumnado parece acostumbrado. Frente a la visión algorítmica que identifica el método científico con una receta casi infalible que produce conocimiento «correcto», la realidad del artículo de investigación es bien diferente. Así, por ejemplo, no es difícil detectar expresiones que expresan duda o incertidumbre (Hyland, 1998). Por ejemplo:

\section{- Los datos parecen indicar que existe una correlación significativa...}

- La elevada concentración está originada, con toda probabilidad, por la presencia de...
- La regulación de la proteína pudiera estar controlada por las moléculas identificadas...

- No se puede descartar la presencia de elementos pesados en la disolución...

- A la vista de la figura 2, la explicación más razonable para los resultados es que...

Parece existir cierto consenso acerca de los objetivos generales que rigen el uso de este tipo de pautas retóricas en los artículos: así, por ejemplo, aunque el empleo de expresiones que reflejan inseguridad o incertidumbre aparentemente debilita una argumentación, en realidad la fortalece al sugerir, en vez de imponer, las conclusiones como la forma más probable de explicar unos resultados obtenidos (Meyer, 1997). Es aconsejable discutir en clase las ventajas que plantea esta estrategia retórica; por ejemplo, sirve como escudo ante posibles errores o interpretaciones alternativas. Se pone, de nuevo, de manifiesto el carácter tentativo del conocimiento científico a la vez que se destaca el uso de las estrategias de persuasión que utilizan los investigadores para tratar de convencer a sus colegas de la validez de sus conclusiones mientras mantienen una prudente reserva, como una especie de cura en salud. Es interesante el contraste que existe entre este rasgo, muy propio de los artículos de investigación, y la prosa científica que se utiliza en los libros de texto, la cual como es sabido, suele dar la impresión de que todo el conocimiento que se presenta en ellos se compone de verdades definitivas.

\section{ANÁLISIS DIDÁCTICO DE ALGUNOS APAR- TADOS CONCRETOS DE LOS ARTÍCULOS DE INVESTIGACIÓN}

\section{La relación de autores y el orden en que éstos apa- recen}

Los nombres y direcciones de los autores de un artículo suelen aparecer inmediatamente después del título. Como hemos indicado, una de las concepciones comunes sobre la ciencia concibe esta actividad como una búsqueda desinteresada de la verdad. Sin embargo, la ciencia también es una profesión con sus propias normas de acceso y promoción y uno de los factores que se toma en consideración en la evaluación de la actividad de los científicos es precisamente la producción de artículos y otros documentos. Por esta razón es tan importante para los investigadores publicar a toda costa o, lo que es lo mismo, conseguir que su nombre aparezca en la mayor cantidad posible de publicaciones de calidad (Campanario, 1999).

Un aspecto interesante de un artículo científico es el orden en el que aparecen los autores cuando son más de uno. Podemos utilizar este elemento como "pretexto» para tratar en clase los factores relacionados con el reconocimiento científico. El orden de los autores depende de múltiples factores y de las costumbres y normas propias del grupo de investigación y del área en que trabajan los 
autores del artículo. A continuación se citan los formatos más habituales:

a) Orden alfabético. Este formato evita los problemas de atribución de los trabajos.

b) El primer autor es el principal. Se supone que el primer autor es el más importante y el resto colabora bajo su dirección.

c) El último autor es el investigador principal. Con este formato se intenta muchas veces otorgar mayor visibilidad a otros miembros del equipo (Merton, 1985).

El análisis de los formatos anteriores permite discutir con el alumnado la importancia de factores aparentemente «extracientíficos» en la construcción del conocimiento, en este caso, el reconocimiento de los autores de una contribución determinada.

\section{Las citas y referencias bibliográficas desempeñan diversas funciones en la retórica y modos de argu- mentar en la ciencia}

Una diferencia notable entre los manuales escolares y los artículos de investigación es que éstos están llenos de citas y referencias a trabajos anteriores. De nuevo, ésta es una característica diferencial que puede servir para introducir una actividad de debate en clase orientada a analizar el papel de la argumentación en la construcción del conocimiento científico. Una parte importante de la argumentación científica consiste, precisamente, en el uso de citas a trabajos y fuentes anteriores.

Aunque todavía no existe una teoría generalmente aceptada sobre los procesos cognitivos de cita, es interesante analizar con el alumnado el papel de las mismas en la construcción del discurso científico porque, incluso sin entender plenamente el contenido de un artículo, es posible elaborar una taxonomía rudimentaria de las supuestas razones que tienen los investigadores para citar otros trabajos. En esta sección destacamos las razones más evidentes. El lector interesado puede consultar los trabajos clásicos de Gilbert (1977) o Cronin (1984) o bien la web del profesor Eugene Garfield, presidente del Institute for Scientific Information (http://garfield.library .upenn.edu), en particular Garfield (1996).

En el aprovechamiento didáctico del estudio de las citas bibliográficas es posible insistir al alumnado en los aspectos siguientes:

a) Una referencia entre dos documentos indica algún tipo de relación conceptual entre los mismos y esto ayuda a situar un trabajo en el marco de un paradigma o teoría.

b) Las citas bibliográficas otorgan autoridad a las afirmaciones y puntos de vista del autor (Gilbert, 1977) (nótese, por ejemplo, cómo se utiliza una cita en la frase anterior para reforzar su contenido). Esta realidad puede resultar sorprendente para los alumnos y alumnas que, ingenuamente, pueden pensar que los científicos se limitan a «descubrir» la verdad sin que sea necesario convencer o argumentar acerca de la validez de dicha verdad.

c) Existen citas negativas en las que se corrigen, matizan o contradicen artículos anteriores propios o de otros investigadores.

e) Los científicos tienen la obligación moral de reconocer los antecedentes de su trabajo, mediante las citas correspondientes. Esta realidad puede servirnos para discutir con el alumnado el tema de la ética científica y de las luchas por la prioridad (Campanario, 1999).

f) Es posible que algún alumno o alumna nos plantee la siguiente cuestión: por qué es raro encontrar citados algunos autores de los contenidos clásicos que aparecen en sus libros de texto (p. e., «la ley de Coulomb»). En este caso se produce otro fenómeno: los contenidos que forman parte del conocimiento común rara vez se citan. Precisamente, la incorporación por obliteración es uno de los mecanismos por los que los nuevos descubrimientos pasan al conocimiento común de manera que pocos científicos sienten la obligación de citar la fuente original donde se describe dicho descubrimiento (Garfield, 1993).

g) Existe otra categoría genérica en las citas y que puede llegar a significar un porcentaje significativo de las referencias incluidas en un artículo. Algunos estudios empíricos lo cifran entre un $20 \%$ y un $40 \%$ (Gilbert, 1977, p. 119; King, 1987).

Los elementos anteriores pueden ayudarnos a clasificar las citas en una taxonomía sencilla, que puede servir para analizar aspectos sociales en la construcción del conocimiento científico a los que rara vez se presta atención. Los alumnos más avanzados pueden intentar realizar una clasificación por sí mismos.

\section{La sección de discusión ilustra de nuevo la necesidad de convencer a otros científicos}

Durante su proceso de escolarización, los estudiantes se enfrentan a muchas afirmaciones que dan a entender implícitamente que los resultados experimentales (o su transformación en tablas y gráficas) permiten descubrir la verdad sin más problemas. Por ejemplo, tal molécula se descompone cuando la temperatura alcanza un determinado valor; el peso molecular de tal compuesto, según el método de X, es tal o cual; o la pendiente de la gráfica $\mathrm{X}$ permite obtener el período del péndulo mediante una simple operación matemática. Dado que rara vez hacemos que el alumnado se enfrente a un artículo de investigación, es difícil que nuestros pupilos se den cuenta de la cantidad notable de argumentaciones y suposiciones que respaldan casi cualquier afirmación que se desprende de los resultados, gráficos o tablas obtenidos en un trabajo «de verdad». Como bien señala Latour, los investigadores pueden dedicar una gran cantidad de tiempo y esfuerzo a aumentar la credibilidad de una frase determinada (Latour, 1987).

La sección de discusión que suele aparecer en cualquier artículo de investigación (a veces esta sección va unida 
a la de resultados) es un buen lugar para descubrir cómo la interpretaciones de los autores del trabajo permiten obtener determinadas conclusiones a partir de los resultados, pero también en función de:

a) la teoría que orienta la investigación;

b) los métodos y procedimientos experimentales utilizados;

\section{c) las limitaciones experimentales.}

La idea básica que deberíamos tratar de reforzar es que cualquier resultado obtenido (una constante física, una nueva sustancia, etc.) es, en esencia, una interpretación de determinadas medidas o procedimientos, a la luz de un paradigma o de un conjunto de conocimientos compartidos por una comunidad. Conviene insistir en este aspecto al alumnado, para dejar claro que, lejos de ser la verdad absoluta evidente por sí misma a partir de unos resultados obtenidos mediante un método fijo, el conocimiento científico es siempre tentativo, fruto de interpretaciones y restricciones y rara vez tenemos certeza total de que no existan otros factores que puedan explicar los datos experimentales.

\section{La sección de agradecimientos ilustra la relevancia de los factores sociales en la ciencia}

La sección de agradecimientos que aparece en casi cualquier artículo puede dar lugar también a interesantes debates entre el profesorado y los estudiantes. Por ejemplo, suelen ser frecuentes los agradecimientos a otros investigadores que colaboraron en determinadas tareas o bien a instituciones académicas o empresas que permitieron la utilización de determinados equipos o facilitaron recursos para la investigación. Esta observación puede permitirnos realizar una breve disquisición sobre la necesidad de colaboración entre científicos como única forma de realizar determinadas investigaciones que requieren el uso de aparatos o métodos tan sofisticados que sólo están disponibles en determinados centros o laboratorios.

Son más comunes los agradecimientos a las agencias e instituciones que financian la investigación. De hecho, una de las condiciones que estas instituciones suelen exigir para conceder fondos es que se citen las ayudas recibidas en cualquier publicación que se derive de los proyectos financiados. De nuevo, esta realidad puede utilizarse en clase como pretexto para abordar el tema de las relaciones ciencia-tecnología-sociedad y discutir el papel de los gobiernos y las políticas científicas en la creación de nuevo conocimiento. En muchas ocasiones las necesidades económicas son determinantes en la financiación de nuevas líneas de investigación. Basta una breve incursión en el Boletín Oficial del Estado (o a las páginas web de los Ministerios de Ciencia y Tecnología (http://www.mcyt.es) y de Educación, Cultura y Deportes (http://www.mecd.es) para comprobar cómo muchas convocatorias de proyectos de investigación están orientadas a líneas específicas y prioritarias de evidente incidencia económica.
La financiación de la investigación por los gobiernos está relacionada también con la necesidad de los departamentos universitarios y de los grupos de trabajo de mantener en funcionamiento toda su estructura. Esta estructura no sólo se refiere a los materiales y recursos, sino que afecta a las personas que realizan su tarea profesional en el seno del grupo. Una de las actividades de los gestores de los equipos de investigación que más tiempo consume consiste precisamente en conseguir becas y ayudas para poder pagar los (magros) salarios de los estudiantes de doctorado y de otros colaboradores que realizan tareas ocasionales (Campanario, 1999). Como puede comprobarse, de nuevo se pone en evidencia una realidad y es que los científicos no son (no pueden ser) seres dedicados únicamente y por entero a la búsqueda de la verdad por la verdad. Si se trabaja en determinadas líneas o temas es más fácil conseguir recursos y, por tanto, generar resultados y obtener publicaciones, con todas las consecuencias que ello tiene, no sólo para el avance del conocimiento, sino para la promoción profesional y la ocupación de puestos de relevancia en la estructura de poder de la ciencia.

Es fácil encontrar otros agradecimientos que hacen referencia a las sugerencias proporcionadas por «asesores anónimos (referees)». Podemos aprovechar la ocasión para explicar al alumnado quiénes son estos referees y por qué su papel es tan importante en el control de calidad de los artículos que se reciben en una revista para su publicación. Para ello es conveniente presentar brevemente el marco general del sistema editorial de la práctica totalidad de las revistas académicas (Campanario, 1998a; Campanario, 1998b; Campanario, 2002a; Weller, 2001). Distinguimos entre:

a) El editor (o persona de reconocido prestigio, responsable de la revista).

b) Un comité editorial (expertos en distintos temas propios del campo de conocimientos de la revista). Este comité editorial suele ser nombrado por el editor.

c) Los asesores ocasionales (o referees) que, en definitiva, son los que evalúan la calidad de los originales que se reciben en la revista.

Que el juicio de estos especialistas puede resultar equivocado lo demuestra el hecho de que al menos 36 ganadores del Premio Nobel tuvieron dificultades con los referees y editores de las revistas, que rechazaron inicialmente los trabajos que les harían merecedores de dicho galardón (Campanario, 2002b). Ciertamente, a veces los científicos son los primeros que se oponen a los nuevos descubrimientos.

\section{Fechas de recepción y aceptación}

Cada vez más revistas académicas publican las fechas de recepción y aceptación de los trabajos recibidos. El origen de esta costumbre tiene que ver con la necesidad de establecer la prioridad en los trabajos científicos (Merton, 1985). Podemos aprovechar estos datos como un pretexto para discutir en clase uno de los problemas del sistema de comunicación científica que más quejas 
suscita: la lentitud de todo el proceso. Como es fácil comprobar por las fechas que acompañan a los artículos publicados, el tiempo que transcurre entre la recepción de un original y su aceptación puede llegar a ser incluso de un año o más debido al tiempo que exige todo el proceso de evaluación por referees. Igualmente, el tiempo que transcurre desde que un artículo es aceptado hasta que aparece impreso puede llegar a ser también de casi un año, dependiendo de las revistas y de las disciplinas (sirva como ejemplo este mismo artículo).

La lentitud en el sistema de comunicación científica puede ayudarnos a ilustrar las enormes precauciones que se toman para evitar errores en las interpretaciones o conclusiones. Está en juego nada menos que la reputación de las revistas académicas, y los editores quieren estar seguros de que los artículos que aparecen en ellas han sido revisados concienzudamente por los referees para evitar problemas. Una consecuencia llamativa de todo el lento proceso anterior es que los artículos que leemos hoy pueden estar basados en estudios realizados hace dos o más años: un retraso notable. En nuestra experiencia previa, este dato, por sí mismo, tiene un impacto notable en las personas que no están familiarizadas con el trabajo científico y que mantienen ideas ingenuas acerca de la mecánica del llamado «descubrimiento» científico.

\section{ALGUNAS SUGERENCIAS CONCRETAS PARA UTILIZAR LOS ARTÍCULOS DE INVESTIGA- CIÓN COMO RECURSO EN LA ENSEÑANZA DE LAS CIENCIAS}

Como se ha indicado más arriba, en este trabajo no planteamos ni pretendemos sugerir que el alumnado analice en profundidad el contenido de un artículo de investigación. Se trata de conseguir, mediante actividades dirigidas y mediante una búsqueda por parte del alumnado, que un medio de comunicación tan fundamental como el artículo de investigación nos permita plantear en clase algunos temas relacionados con la dinámica de la ciencia y con la construcción del conocimiento científico.

A continuación se ofrecen algunas sugerencias concretas para utilizar este recurso en clase. Los profesores y profesoras interesados en utilizar este recurso seguramente podrán diseñar actividades adaptadas a las necesidades y características propias de su alumnado.

a) Tal vez la actividad más sencilla consista en una simple discusión en clase sobre algún aspecto concreto que se desee abordar. Esta discusión puede ir seguida de una tarea de análisis de un artículo de investigación determinado. Como en cualquier otra tarea de clase, puede ser conveniente organizar esta actividad en grupos.

b) Otra posibilidad puede consistir en plantear una tarea de localización o comentario por escrito de determinados aspectos del artículo que el profesor o profesora ha señalado previamente. Por ejemplo, se puede pedir a los alumnos que comenten o localicen ejemplos de expresiones que demuestren incertidumbre o dudas acerca de determinados resultados. c) Otra alternativa consiste en utilizar noticias aparecidas en los medios de comunicación que hagan referencia a aspectos relacionados con la ciencia, para plantear el análisis de un artículo de investigación. Por ejemplo:

- Noticias que hagan referencia a investigaciones promovidas por tal o cual institución. Estas noticias nos permiten abordar el tema de la financiación de la investigación científica y cómo se refleja este aspecto en la sección de agradecimientos.

- Noticias en las que se utilicen expresiones como «nuevo método», «desconocido hasta ahora» permiten plantear temas en calidad de novedad, elemento que contribuye a dar valor a un trabajo de investigación. En este caso se trataría de identificar expresiones en un artículo científico que hagan referencia a dicha novedad.

- Noticias que citen trabajos realizados por «un equipo» de tal o cual país. Estas noticias nos permiten abordar temas como la organización de la investigación científica, el orden de los autores en un trabajo científico y la atribución del crédito.

- Noticias en las que se haga referencia al «método científico» o se indique que algo ha sido «comprobado científicamente». En este caso podemos plantear en clase la necesidad de profundizar en las visiones inadecuadas acerca de la ciencia y el supuesto «método científico», comparando lo que se dice en muchos libros de texto acerca de este supuesto «método científico» y lo que se deduce al analizar un artículo de investigación. Estas noticias pueden servirnos como pretexto para abordar otro tema que hemos comentado más arriba: las limitaciones de los métodos que se utilizan en la investigación científica, algo que suele señalarse de manera explícita en los artículos de investigación y que los propios alumnos pueden encargarse de detectar.

d) Una posibilidad interesante consiste en preparar una selección de materiales obtenidos de diferentes artículos. A veces resulta complicado encontrar un artículo relativo a un tema concreto en el que sea posible identificar fácilmente todos los aspectos que hemos tratado en este trabajo. Con una selección debidamente organizada y señalada (por ejemplo, subrayando las partes de interés), se pueden abordar los temas que se deseen.

e) Tareas de clasificación en una taxonomía sencilla, por ejemplo, de los tipos de citas y referencias que se incluyen en un artículo, de acuerdo con el contexto de la cita (cita neutral, cita que apoya las conclusiones propias, citas negativas a otros trabajos previos...).

f) Tareas de comparación entre las características del lenguaje científico que se utiliza en los libros de texto con el que se usa en los artículos de investigación. Por ejemplo, como se ha indicado más arriba, en los libros de texto rara vez aparecen ejemplos de incertidumbre o de afirmaciones tentativas. Casi siempre el contenido se presenta como una verdad definitiva, en abierto contraste con las estrategias argumentativas utilizadas en los artículos de investigación. 
g) Con el fin de facilitar la tarea de cuestionamiento de ideas inadecuadas sobre la ciencia y el conocimiento científico, se puede pedir al alumnado que exprese su grado de acuerdo o desacuerdo con determinadas afirmaciones sobre el conocimiento científico (p. e., «el conocimiento científico es conocimiento probado más allá de toda duda»). A continuación se presentan ejemplos que contradicen dichas afirmaciones y se pide a los estudiantes que piensen sobre el problema que se plantea ante dicho conflicto.

h) Tarea de comparación de artículos de diferentes disciplinas. Por ejemplo, es útil contrastar el número de referencias bibliográficas en artículos de matemáticas con el número de referencias bibliográficas en artículos de bioquímica y relacionar estas referencias con el carácter más o menos experimental de ambas disciplinas.

Las sugerencias anteriores, junto con las directrices generales que se presentan en el anexo, proporcionan cierta orientación sobre las posibilidades didácticas de los artículos de investigación como un recurso que puede ayudar al profesorado a plantear discusiones y análisis en clase sobre los procesos de construcción y evaluación del conocimiento científico.

\section{CONCLUSIONES}

Resulta sorprendente el poco uso que hacemos en clase de recursos docentes alternativos que son fáciles de utilizar y están disponibles en cantidades casi ilimitadas en las bibliotecas de cualquier universidad española o en internet. La realidad anterior resulta todavía más llamativa si tenemos en cuenta que quienes nos dedicamos a la enseñanza de las ciencias somos físicos, químicos, biólogos o geólogos. Por tanto, contamos con una formación que debería permitirnos utilizar alternativas imaginativas en vez del recurso inmediato a los libros sobre filosofía de la ciencia a la hora de indagar en los procesos de construcción del conocimiento científico.

De la discusión realizada en las secciones anteriores se desprenden algunas conclusiones sobre la posibilidad de utilizar los artículos de investigación en la enseñanza de las ciencias. En primer lugar, el uso de este recurso estaría orientado a conseguir que el alumnado desarrolle una visión menos simplista del trabajo científico, a la vez que se cuestionan concepciones epistemológicas inadecuadas sobre los procesos de construcción y justificación del conocimiento. Mediante una búsqueda guiada de determinadas características y elementos que pueden encontrarse en un artículo de investigación, es posible descubrir que su elaboración, su estructura y recursos argumentativos ponen de manifiesto factores sociales a los que rara vez se presta atención, a la vez que contradicen abiertamente algunas suposiciones ingenuas muy extendidas acerca de la ciencia y el conocimiento científico. Insistimos en que no planteamos un análisis del contenido de un artículo científico (algo que queda fuera del alcance de la mayor parte del profesorado), sino un debate en torno a ciertas características formales de los artículos científicos (p. e., citas, determinadas expresiones, agradecimientos, etc).

Cuando un estudiante se inicia en el trabajo científico en un laboratorio, se da cuenta de que los experimentos «reales» son más complicados que los que se describen en su libro de texto. El neófito descubre, por ejemplo, que existen factores como rozamientos, calentamientos, instrumentos con agujas que oscilan, pérdidas, interferencias, imprecisiones instrumentales y otras «molestias» a las que es necesario prestar casi tanta atención como al fenómeno en sí que se estudia. Incluso, cuando un científico novel se inicia en la investigación, tiene dificultades para pasar de la relativa seguridad en que se habían desarrollado sus prácticas de laboratorio, a la incertidumbre notable que es inherente casi siempre a la tarea de indagación en la ciencia (Delamont y Atkinson, 1991). De la misma manera, el primer contacto con un artículo académico pone de manifiesto que los procesos de justificación del conocimiento científico, las estrategias argumentativas, los factores sociales y la implicación personal de los investigadores desempeñan un papel fundamental a la hora de convencer a los hipotéticos lectores $y$, por tanto, al resto de la comunidad académica.

Podemos comparar la situación anterior con la estructura de las prácticas de laboratorio tradicionales que, en la mayoría de los casos, es el único trabajo científico al que nuestros alumnos y alumnas suelen tener acceso. En las prácticas de laboratorio tradicionales, los datos y tablas suelen hablar por sí mismos sin que sea necesario argumentar ni convencer a nadie. El uso generalizado de esta forma de trabajo puede reforzar visiones inadecuadas sobre la construcción del conocimiento científico y sobre el estatus del mismo como algo que debe su «veracidad» a unos datos que se han obtenido aplicando un método determinado.

Creemos que la propuesta que realizamos merece explorarse con más profundidad, al menos en los niveles de enseñanza universitaria y, ocasionalmente, bachillerato. Entre las ventajas previsibles de nuestra propuesta destacamos las siguientes:

a) El coste de los nuevos materiales a utilizar es irrisorio.

b) Se fomentan los enfoques multidisciplinares. Como se indica más arriba, investigadores ubicados en áreas como sociología o lingüística prestan cada vez más atención a los procesos sociales de construcción del conocimiento científico. Por tanto, algunas de las tareas que proponemos se prestan a una interesante colaboración entre el profesorado de lengua, filosofía y ciencias.

c) Las propuestas que formulamos en este trabajo abren nuevas líneas de indagación y actuación en el aula para el profesorado investigador que tenga interés en mejorar su docencia. Estas nuevas líneas pueden dar lugar a desarrollos ulteriores sobre la forma de utilizar los artículos de investigación como un recurso docente más.

d) Con el enfoque que proponemos, se abordan contenidos relacionados con la retórica científica a los que rara 
vez se presta atención, a pesar de la importancia que se concede en los últimos años a los aspectos relacionados con la construcción y justificación del conocimiento.

e) Se proporciona al alumnado una oportunidad de tener otro tipo de experiencias científicas. Al igual que nos parece necesario que los estudiantes accedan al laboratorio, que tomen contacto con los aparatos y que hagan ciencia (aunque sea en miniatura), creemos que es ineludible también que tengan acceso a la parte más «blanda» de la construcción del conocimiento científico: la comunicación entre investigadores y las estrategias de razonamiento y argumentación que se utilizan en las disciplinas académicas.

f) Algunas de las propuestas que se formulan aquí pueden dar lugar a pequeñas investigaciones no sólo por parte de alumnos universitarios de ciencias, sino también por parte de estudiantes de otras carreras o por profesores y profesoras que tengan interés en la dinámica de la ciencia. g) Ésta es una oportunidad para que el profesor o profesora aprendan. Dado que, con el recurso que proponemos, se presta atención a aspectos poco conocidos (incluso muchas veces para los propios científicos), se exige del docente un cierto esfuerzo de formación y actualización que está sobradamente compensado por el interés y novedad de los contenidos que se aprenden.

\section{AGRADECIMIENTOS}

Este trabajo se realizó en el marco de la acción PR2002-0046, financiada por el Ministerio de Educación, Cultura y Deportes para realizar una estancia de investigación en la Universidad de Wollongong (Australia). Quiero expresar mi agradecimiento a Brian Martin y Soon Nam Kim, de dicha universidad, por las estimulantes discusiones que mantuvimos sobre el sistema de publicación científica y a dos revisores anónimos que formularon sugerencias que han ayudado a mejorar el trabajo. 


\section{REFERENCIAS BIBLIOGRÁFICAS}

CAMPANARIO, J.M. (1998a). Peer review for journals as it stands today. Part 1. Science Communication, 19, pp. 181211 .

CAMPANARIO, J.M. (1998b). Peer review for journals as it stands today. Part 2. Science Communication, 19, pp. 277-306.

CAMPANARIO, J.M. (1999). La ciencia que no enseñamos. Enseñanza de las Ciencias, 17, pp. 397-410.

CAMPANARIO, J.M. (2002a). El sistema de revisión por expertos (peer review): muchos problemas y pocas soluciones. Revista Española de Documentación Científica, 25, pp. 166-184.

CAMPANARIO, J.M. (2002b). Rejecting Nobel class papers and resisting Nobel class discoveries. (En revisión). Disponible en <http://www.uah.es/otrosweb/jmc>.

CAMPANARIO, J.M. (2002c). La enseñanza de las ciencias en preguntas y respuestas. Universidad de Alcalá: Alcalá de Henares. Disponible en <http://www.uah.es/otrosweb/jmc>.

CAMPANARIO, J.M. (2003). Científicos que cuestionan paradigmas dominantes: implicaciones para la enseñanza de las ciencias. (En revisión).

CAMPANARIO, J.M. y MARTÍN, B. (2003). Challenging dominant Physics paradigms. (En revisión).

CAMPANARIO, J.M. y OTERO, J.C. (2000). Más allá de las ideas previas como dificultades de aprendizaje: las pautas de pensamiento, las concepciones epistemológicas y las estrategias metacognitivas de los alumnos de ciencias. Enseñanza de las Ciencias, 18, pp. 155-169.

CHALMERS, A.F. (1982). ¿Qué es esa cosa llamada ciencia? Madrid: Siglo XXI Editores.

CHINN, C.A. y BREWER, W.F. (1993). The role of anomalous data in knowledge acquisition: A theoretical framework and implications for science instruction. Review of Educational Research, 63, pp. 1-49.

COLLINS, H.M. (2001). Tacit Knowledge, Trust and the Q of Sapphire. Social Studies of Science, 31, pp. 71-85.

COLLINS, H. y PINCH, T. (1993). The Golem: What you should know about science. Cambridge: Cambridge University Press.

CRONIN, B. (1984). The citation process. Londres: Taylor Graham.

DELAMONT, S. y ATKINSON, P. (2001). Doctoring Uncertainty: Mastering Craft Knowledge. Social Studies of Science, 31, pp. 87-107.

FERNÁNDEZ-CANO, A. (1999). Producción educativa española en el Social Index Citation Indez (1988-97). Revista Española de Pedagogía, 214, pp. 509-524.

FEYNMAN, R.P. (1965). The development of the space-time view of quantum electrodynamics. Nobel Lecture, 11. Disponible en <http://www.nobel.se>.

GARFIELD, E. (1993). To what extent is the research literature cited? Lowell Hargens and David Bott examine citation rates in sociology. Current Contents, 5, pp. 3-4. Disponible en <http://garfield.library.upenn.edu>.

GARFIELD, E. (1996). When to cite. Library Quarterly, 66, pp. 449-458. Disponible en <http://garfield.library.upenn.edu>.

GASKELL, P. J. (1992). Authentic science and school science. International Journal of Science Education, 14, pp. 265-272.

GILBERT, G.N. (1977). Referencing as persuasion. Social Studies of Science, 7, pp. 113-122.

HYLAND, K. (1998). Hedging in scientific research articles. Amsterdam: John Benjamins.

HYLAND, K. (2002). Directives: Argument and engagement in academic writing. Applied Linguistics, 23, pp. 215-239.

KING, J. (1987). A review of bibliometric and other science indicators and their role in research evaluation. Journal of Information Sciences, 13, pp. 261-276.

KUHN, T. (1971). La estructura de las revoluciones científicas. México: Fondo de Cultura Económica.

LATOUR, B. (1987). Science in action. Cambridge, Massachusetts, EEUU: Harvard University Press.

MERTON, R.K. (1985). La sociología de la ciencia. Madrid: Alianza Universidad.

MEYER, P. G. (1997). Hedging strategies in written academic discourse: stregthening the argument by weakening the claim, en Raija Markkanen y Hartmut Schröder (eds.). Hedging and discourse. Approaches to the analysis of a pragmatic phenomenon in academic texts. Berlín: Walter de Gruyter.

OTERO, J.C. (1985). Assimilation problems in traditional representations of scientific knowledge. European Journal of Science Education, 7, pp. 361-369.

POLANYI, M. (1958). Personal knowledge. Londres: Routledge \& Kegan Paul.

RYAN, A.G. y AIKENHEAD, G.S. (1992). Students' preconceptions about the epistemology of science. Science Education, 76, pp. 559-580.

STRUBE, P. y LYNCH, P. P. (1984). Some influences on the modern science text: Alternative science writing. European Journal of Science Education, 6, pp. 321-338.

WELLER, A. C. (2001). Editorial peer review. Its strengts and weaknesses. Nueva York: ASIST Monograph Series.

WOLFF-MICHAEL, R. (1994). Physics students' epistemologies and views about knowing and learning. Journal of Research in Science Teaching, 31, pp. 5-30. 


\author{
ANEXO \\ Guía para el profesorado de ciencias interesado en utilizar los artículos de investigación \\ como un recurso docente
}

Las orientaciones que se presentan en este apartado tienen como objetivo facilitar el trabajo didáctico con los artículos de investigación. Es posible que algunas de las ideas que aquí se discuten sólo puedan desarrollarse plenamente con estudiantes de nivel universitario.

\title{
ASPECTOS GENERALES
}

\section{a) El artículo científico como medio de comunicación}

- Se plantea la necesidad de tener en cuenta los procesos de comunicación como elemento imprescindible en la construcción del conocimiento científico.

- Se presenta el sistema de comunicación científica (revistas científicas, congresos, internet).

- Se aborda el papel de la comunidad científica como juez último de los nuevos descubrimientos y propuestas.

- Implicaciones que tiene para los científicos la publicación académica (reconocimiento, promoción, prioridad...).

- Productividad de los científicos: se presentan algunas cifras sobre el número de artículos que producen los científicos. A diferencia de lo que sucede en otros terrenos (p. e., periodismo), los investigadores producen muchos menos trabajos.

\section{b) Tipos de artículos}

- Se identifican algunos de los tipos de artículos científicos que se pueden encontrar en revistas especializadas

\section{c) Estructura de los artículos de investigación}

- Se analiza la división en apartados de un artículo de investigación (título, autores, resumen (abstract), palabras clave (keywords), introducción, objetivos, métodos, resultados, discusión, conclusiones, agradecimientos, referencias, anexos).

- Se presta especial atención a los apartados que pueden resultar más desconocidos para el alumnado (resumen (abstract), palabras clave [keywords], discusión, referencias).

- Se discute si la estructura de un artículo de investigación es compatible con las visiones simplistas acerca del método científico, tal como se describe en muchos libros de texto.

- Con alumnos de nivel universitario puede plantearse una breve discusión sobre las visiones falsacionistas acerca de la construcción del conocimiento científico. ¿Es posible identificar intentos por falsar teorías o, más bien, se trata de confirmarlas?

\section{d) Temas de los artículos de investigación}

- Se llama la atención sobre el carácter limitado de los temas que se abordan en un artículo de investigación.

- Se insiste en el papel del marco general (paradigma o teoría) que da sentido a la investigación realizada.

- Se analizan las argumentaciones que ofrecen los autores de artículos científicos para justificar el valor de su trabajo de investigación.

- Se analizan las conclusiones del artículo para comprobar que, lejos de afirmar que el trabajo realizado es definitivo, con frecuencia los autores insisten en el carácter tentativo del mismo y en la posibilidad de nuevos estudios e investigaciones.

- Se analizan los indicios en el artículo que hacen referencia a la novedad del trabajo realizado.

- Se discuten las llamadas de atención acerca de las limitaciones de los resultados obtenidos o de los métodos experimentales utilizados. 
e) El estilo del lenguaje científico utilizado en un artículo de investigación

- Se analizan los indicios y las expresiones que utilizan los autores para guiar al lector en su argumentación y planteamiento del tema.

- Se comparan estos indicios y expresiones con las ideas típicas acerca de la supuesta neutralidad del lenguaje científico.

- Se comparan los estilos de lenguaje científico que aparecen en los libros de texto de ciencias y en un artículo de investigación. En particular, se insiste en las diferencias en cuanto a las expresiones que denotan incertidumbre o conclusiones tentativas frente a la forma tradicional de presentar contenidos científicos en libros de texto como si se tratase de verdades finales.

- Se analizan las llamadas de atención de los autores del artículo sobre el interés o la relevancia de su trabajo.

- Se analizan algunas estrategias argumentativas orientadas a convencer al lector de que determinadas interpretaciones deben descartarse. Se aprovecha para insistir en el importante papel de la argumentación y de la teoría a la hora de interpretar los resultados experimentales obtenidos.

\section{APARTADOS CONCRETOS}

\section{f) Autores del trabajo y orden en que aparecen}

- Se abordan los aspectos sociales derivados del reconocimiento científico y del sistema de recompensas en la ciencia. Se insiste en que los científicos buscan, fundamentalmente, el reconocimiento del resto de la comunidad investigadora.

- Se plantea el papel de la publicación en el reconocimiento académico («publica o mиere»).

\section{g) Citas y referencias y su papel en la argumentación científica}

- Se llama la atención sobre las referencias bibliográficas que aparecen al final del artículo.

- Se analiza la relación entre las citas que aparecen en el texto y las referencias bibliográficas que se incluyen al final del artículo.

- Se discute la importancia de las citas como elementos que contribuyen a fundamentar el trabajo realizado.

- Se plantean taxonomías sencillas para clasificar citas bibliográficas. Se identifican ejemplos de citas mediante las que se busca apoyo para una argumentación, mediante las que se reconocen antecedentes previos o mediante las que se promociona el trabajo propio anterior. Se intenta identificar algún ejemplo de cita negativa.

- Con estudiantes avanzados puede destacarse el papel de las referencias en la sección de métodos para centrar el ámbito y la orientación del trabajo.

\section{h) La sección de discusión}

- Se analiza el papel de esta sección en la argumentación general que se plantea en el trabajo.

- Se insiste en la necesidad de comentar y analizar los datos y resultados obtenidos en la investigación. Probablemente muchos estudiantes piensen que los datos y resultados son evidentes por sí mismos. La mera existencia de una sección de «discusión» demuestra que es precisa una labor de análisis para dar sentido a dichos datos y resultados.

- En esta sección suelen ser más frecuentes los ejemplos de frases que indican que los resultados que se obtienen son tentativos o admiten interpretaciones alternativas (que se analizan y se descartan) o plantean la necesidad de realizar investigaciones posteriores.

\section{i) Otros apartados y secciones}

- La sección de agradecimientos permite plantear un debate sobre la financiación de la investigación científica, sobre el carácter colectivo de la misma y sobre las redes de investigadores que intercambian información, materiales y resultados.

- Si aparecen las fechas de recepción y aceptación de un artículo, es posible analizar con los alumnos el sistema de publicación académica y el papel de la revisión de los trabajos por expertos y asesores con el fin de asegurar la calidad de la tarea de investigación que se realiza. 